\title{
Numerical simulation for the steady nanofluid boundary layer flow over a moving plate with suction and heat generation
}

\author{
M. Ferdows ${ }^{1} \cdot$ MD. Shamshuddin ${ }^{2}$ D $\cdot$ S. O. Salawu ${ }^{3} \cdot$ K. Zaimi $^{4}$
}

Received: 23 June 2020 / Accepted: 15 January 2021 / Published online: 3 February 2021

(c) The Author(s) $2021 \quad$ OPEN

\begin{abstract}
In the study, the steady, laminar, incompressible, convective flow of a viscous fluid over a moving plate is investigated theoretically by adopting different types of nanoparticles. Radiation, internal heat generation and viscous dissipation effects are considered in the energy modeled equation. The governing flow equations for the momentum and temperature are reduced to dimensionless form via similarity transformations. The solutions to the resultant equations alongside with the transformed boundary conditions are numerically obtained using MATLAB package bvp4c. Validation with earlier studies are done for the non-internal heat generation case for two distinct nanoparticles of type Cu-water and Al-water. Extensive visualization of flow rate and heat distributions for various emerging parameters are examined. Temperature is consistently enhanced with a rising Eckert number of both types of nanofluids, whereas it is strongly reduced with rising values of radiation term. Heat transfer coefficient is consistently increased with a nanoparticle volume fraction of high convective heat in the medium.
\end{abstract}

Keywords Nanofluid $\cdot$ Moving plate $\cdot$ Suction $\cdot$ Internal heat generation $\cdot$ Viscous dissipation $\cdot$ bvp4c

$\begin{array}{ll}\text { Abbreviations } \\ C & \text { Constant } \\ C_{f} & \text { Skin friction coefficient } \\ E C & \text { Eckert number } \\ k_{f} & \text { Thermal conductivity of fluid }(\mathrm{W} / \mathrm{m} \mathrm{k}) \\ k_{n f} & \text { Thermal conductivity of nanofluid }(\mathrm{W} / \mathrm{m} \mathrm{k}) \\ \mathrm{ks} & \text { Thermal conductivity of solid fraction }(\mathrm{W} / \mathrm{m} \mathrm{k}) \\ \mathrm{Nr} & \text { Radiation parameter } \\ \mathrm{Nu} & \text { Local Nusselt number } \\ \mathrm{Pr} & \text { Prandtl number } \\ \mathrm{qr} & \text { Radiative heat flux } \\ \mathrm{Re}_{x} & \text { Local Reynolds number } \\ \mathrm{S} & \text { Local suction parameter }\end{array}$

$T$

$T_{w}$

$T_{\infty}$

$u$

$U_{w}$

$V_{o}$

V

$x, y$
Temperature of the field in the boundary layer (K)

Wall temperature of the fluid $(K)$

Temperature of the fluid in free stream (K)

Dimensionless velocity component in $\mathrm{X}$-direction $(\mathrm{m} / \mathrm{s})$

Surface velocity along $x$-axis

Surface velocity along $y$-axis

Dimensionless velocity component in y-direction $(\mathrm{m} / \mathrm{s})$

Distance along and perpendicular to the plate

(m)

M. Ferdows, M. D. Shamshuddin, S. O. Salawu and K. Zaimi authors have contributed equally for the manuscript.

$\triangle$ MD.Shamshuddin, shamshuddin_md@vaagdevi.edu.in; shammaths@gmail.com; M. Ferdows, ferdowsmohammad@yahoo.com; S. O. Salawu, salawu.sulyman@Imu.edu.ng; kunlesalawu2@gmail.com; K. Zaimi, Khairy@unimap.edu.my|'Research Group of Fluid Flow Modeling and Simulation, Department of Applied Mathematics, University of Dhaka, Dhaka 1000, Bangladesh. ${ }^{2}$ Department of Mathematics, Vaagdevi College of Engineering (Autonomous), Telangana State, Warangal, India. ${ }^{3}$ Department of Physical Science, Mathematics Programme, College of Pure and Applied Sciences, Landmark University, Kwara State, Omu-aran, Nigeria. ${ }^{4}$ Department of Mathematics, Institute of Engineering Mathematics, Universiti Malaysia Perlis, Kangar, Malaysia. 


\section{Greek symbols}

$\alpha_{n f} \quad$ Thermal diffusivity of nanofluid $\left(\mathrm{m}^{2} / \mathrm{s}\right)$

$\rho_{f} \quad$ Reference density of fluid fraction $\left(\mathrm{kg} / \mathrm{m}^{3}\right)$

$\rho_{n f} \quad$ Effective density of nanofluid $\left(\mathrm{kg} / \mathrm{m}^{3}\right)$

$\rho_{s} \quad$ Reference density of solid fraction $\left(\mathrm{kg} / \mathrm{m}^{3}\right)$

$\left(\rho c_{p}\right)_{n f}$ Heat capacitance of nanofluid $\left(\mathrm{J} / \mathrm{m}^{3} \mathrm{~K}\right)$

$\mu_{f} \quad$ Dynamic viscosity of fluid $(\mathrm{kg} / \mathrm{m} \mathrm{s})$

$\mu_{n f} \quad$ Dynamic viscosity of nanofluid $(\mathrm{kg} / \mathrm{m} \mathrm{s})$

$\eta \quad$ Similarity variable

$\lambda \quad$ Velocity slip parameter

$\phi \quad$ Solid volume fraction

$\psi \quad$ Stream function

\section{Subscripts}

w Surface conditions

$\infty \quad$ Conditions far away from the plate

nf Nanofluid

\section{Introduction}

In polymer fabrication at high temperature [1, 2], radiative heat distribution takes place in addition to heat convection and heat conduction. The solution of integrodifferential equation of heat radiation is most general, computationally it is very challenging. In the presence of other effects such as magnetohydro-dynamics, rheology are the effortless pragmatic approach that frequently features an approximation algebraic flux that can be of the Traugott P1 flux model, Milne-Eddington type, Rosseland type, six flux Hamaker formulation, Schuster-Schwartzchild type etc. In coating flows of multi-physical, high radiation optical thicknesses are practically and correctly presented using the Rosseland model. Though it does not permit the stimulation of spectal effects or optical viscosity, but provides an evaluating mechanism for the relative role of heat flux radiation and heat conduction. Numerous scholars have investigated radiative heat transfer in materials processing including Shamshuddin et al. [3] (bio convection nanofluid), Kadir et al. [4] (thermal stress analysis), Liu et al. [5] (multi laser processing) and Yue and Reitz [6] (internal combustion energies).

In recent years, nanofluids have been increasingly deployed in industrial and technological systems. They constitute a unique subset of nanomaterials. ethylene glycol, engine oil and water are common fluids that constrained heat transfer due to their low heat conductivity capacity, while metals have remarkably heat conductivities strength than those fluids. Therefore, scattering high thermal conductivity solid particles augments heat conductivity of the subsequent fluid suspension. The idea of nanoliquids was initiated by Choi et al. [7] at Argonne Energy Lab, USA. As noted, thermal conductivity is the magnificent attribute of nanofluids [8]. Carbides (SiC), Nitrides (AIN, SiN), Metallic oxides (Al2O3, TiO2), Metallic $(\mathrm{Al}, \mathrm{Cu})$ or nanotubes carbon with diameter ranges from 10 to $100 \mathrm{~nm}$ are the nanoparticles used in synthesis of nanofluids. Hamid et al. [9] studied transient convective radiation of nanofluid flow past a vertical medium. It was observed that changing in the shape of nanoparticle encourages the rate of heat transfer. Pandey and Kumar [10] investigated Cu-water nanoliquid heat transfer in a moving cylinder boundary layer with slip condition. Many robust mathematical formualtions have been developed for flow of nanoparticles through demanding foundational experimental data. Amanullah et al. [11] examined computationally, the non-Newtonian nanofluid with different nanoparticles. Anwar et al. [12] studied experimentally, the drilling lubricant fluids with nano particles and Bio convection lubricants [13]. Several researchers have studied nanofluid flow with different nanoparticles applying different geometries which has applications in material processing including Shamshuddin et al. [14] (squeezing flow), Anwar Beg et al., [15] (magnetic nanoploymer flow), Rashidi et al. [16] (triangular obstacle), Akar et al. [17] (rotating cylinder), Hamid et al. [18] (duct equipped with porous baffels), Eid [19] (Riga surface), Eid and Mabood [20] (cross nano material flow), Eid and Mabood [21] (micropolar dusty CNTs), Mohebbi et al., [22] (extended surface channel), Mohebbi nd Rashidi [23] (L-shaped heating obstacle enclosure) and Armaghani et al. [24] (baffled L-shaped caviity).

The present article is inspired to provide a more broad understanding of the processes involving heat generating at high temperature. A mathematical equation is formulated for viscous radiative flow fluid from a flat plate with heat source. This formulated equation strengthens the former report of Gangadhar [25] to examine the transfer of thermal radiation and IHG impacts. The modelled partial derivative boundary value equation reduces to an ordinary non-linear derivative boundary value equation with applicable transformation variables. A vigorous numerical solution is gotten with the uses of MATLAB package bvp4c and validation with the non-IHG case Gangadhar [25] is considered. A complete parametric analysis of the impact of suction, velocity slip, Eckert number and thermal radiation on the velocity magnitude, temperature component and skin friction coefficients results are conducted with graphical representation.

\subsection{Mathematical model}

Examine the flow of conducting viscous incompressible liquid of heat transport over a flat moving plate with magnetic effect been ignored. We consider a Cartesian system of coordinate such that the plate corresponds with the $x y$-coordinate and the liquid fills the space 
$z \geq 0$ as defined in Fig. 1. Taken $u=U_{w}(x)$ while $v=V_{0}(x)$ describe the plate velocities in the direction of $x, y$. The fluid velocity is maintained with velocity $U$, and Velocity of the far field and the flat sheet are assumed as $U_{\infty}, U_{w}$ respectively. It is considered that plate temperature is $T_{w^{\prime}}$ while free stream uniform temperature is $T_{\infty}$.

Under the specified assumptions, the associated equations defining the viscous two-dimensional fluid momentum are stated as an extension of the model presented by Gangadhar [25] with IHG and radiative heat flux terms, leading to:

Continuity Equation:

$\frac{\partial u}{\partial x}+\frac{\partial v}{\partial y}=0$

Momentum Equation:

$u \frac{\partial u}{\partial x}+v \frac{\partial u}{\partial y}=v_{n f} \frac{\partial^{2} u}{\partial y^{2}}$

Energy Equation: $u \frac{\partial T}{\partial x}+v \frac{\partial T}{\partial y}=\alpha_{n f} \frac{\partial^{2} T}{\partial y^{2}}-\frac{1}{\left(\rho C_{p}\right)_{n f}} \frac{\partial q_{r}}{\partial y}+\frac{\mu_{n f}}{\left(\rho C_{p}\right)_{n f}}\left(\frac{\partial u}{\partial y}\right)^{2}+\frac{q^{\prime}}{\left(\rho C_{p}\right)_{n f}}$,

Here, $u$ and $v$ denote the component of velocities in the $x$ and $y$ axes. $\rho c_{p}$ is the nanoliquid heat capacity. $q_{r}=-\frac{4 \sigma^{*}}{3 k^{*}} \frac{\partial T^{4}}{\partial y}$ is net radiative heat flux, expanding $T^{4}$ using Taylor's series assuming temperature difference and higher order terms neglecting, thus

$T^{4} \cong 4 T_{\infty}^{3} T-3 T_{\infty}^{4}$

Boundary conditions are subjected to be:

$\left.\begin{array}{ll}u=U_{w}, v=v_{0}, T=T_{w} & \text { at } y=0 \\ u=U_{\infty}, & T=T_{\infty} \text { as } y \rightarrow \infty\end{array}\right\}$

The nanofluid modified properties are simulated according to Gangadhar [25], the nanoparticles thermophysical characteristics and the base liquid are given in the Table 1.

$\left.\begin{array}{l}\alpha_{n f}=\frac{k_{n f}}{\left(\rho C_{p}\right)_{n f}}, \rho_{n f}=\rho_{f}-\phi \rho_{f}+\phi \rho_{s}, \mu_{n f}=\frac{\mu_{f}}{(1-\phi)^{2.5}} \\ \left(\rho C_{p}\right)_{n f}=\left[\left(\rho C_{p}\right)_{f}-\phi\left(\rho C_{p}\right)_{f}\right], k_{n f}=k_{f}\left\{\frac{\left(k_{s} / k_{f}\right)+(n-1)-(n-1) \phi\left(1-\left(k_{s} / k_{f}\right)\right)}{\left(k_{s} / k_{f}\right)+(n-1) \phi\left(1-\left(k_{s} / k_{f}\right)\right)}\right\}\end{array}\right\}$

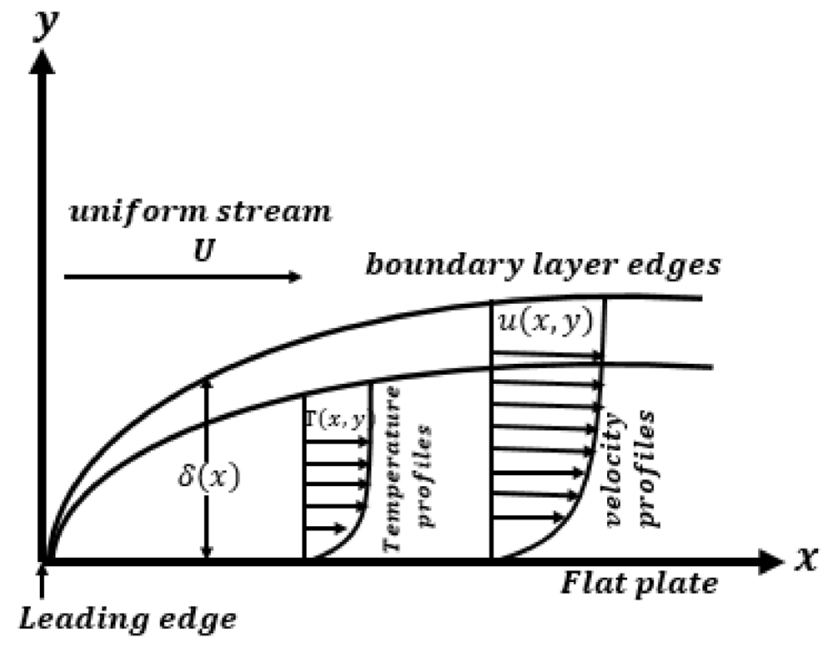

Fig. 1 Boundary layer of 2D laminar flow
Table 1 Nanoparticle thermophysical properties and the base liquid at $20^{\circ} \mathrm{C}(293 \mathrm{~K})$

\begin{tabular}{llll}
\hline Thermo physical & Base Fluid & Nanoparticles & \\
\cline { 3 - 4 } properties & $\mathrm{H}_{2} \mathrm{O}$ (water) & $\mathrm{Cu}$ (Copper) & $\mathrm{Al}_{2} \mathrm{O}_{3}$ (Alumina) \\
\hline$\rho\left(\mathrm{Kgm}^{-3}\right)$ & 997.1 & 8933 & 3970 \\
$C_{p}\left(\mathrm{Jg}^{-1} \mathrm{~K}^{-1}\right)$ & 4179 & 385 & 765 \\
$k\left(\mathrm{Wm}^{-1} \mathrm{~K}^{-1}\right)$ & 0.613 & 401 & 40 \\
\hline
\end{tabular}




\subsection{Transformations}

We have to reframe the boundary layer equations to a non-dimensional system of equations using similarity variable. The objective of similarity conversion is to reduce the independent variable in the governing equations by quantities of transformation. The parlance quantities is worn because of the widening of the boundary layer with distance $x$ from the major edge, both velocity and temperature profile remain geometrically similar $[25,26]$. Similarity variable of the form $[27,28]$;

Here,
$C$ mentioned in Eq. (11) must be constant and independent on $x$ and it is defined as $C=\left[e^{n} / k_{n f} \operatorname{Re}_{x}\left(T_{w}-T_{\infty}\right)\right]$ which is the scale of the strength of the internal heat generation (IHG). Furthermore, to have similarity solution for ordinary differential equations, the terms $\lambda$. $S$ is independent of $x$. These conditions are gratified when $\lambda \propto x^{1 / 2}$ and $v_{0} \propto x^{-1 / 2}$ i.e., $\lambda=A x^{1 / 2} v_{0}=B x^{-1 / 2}$ Where $A, B$ are constants. The terms are the local similarity variables which are used to obtain the dimensionless equations. This gives an invariant transformation that has been adopted by several researches including magnetofluid dynamics and heat transfer. It gives the required

$\left.\psi(x, y)=\left(U_{\infty} v_{n f} x\right)^{1 / 2} f(\eta), \theta=\frac{T-T_{\infty}}{T_{w}-T_{\infty}}, \quad \eta=y\left(\frac{U_{\infty}}{v_{n f} x}\right)^{1 / 2}, S=v_{o}(x) \sqrt{\frac{x}{v_{n f} U_{\infty}}}\right\}$

where $\psi(x, y)$ is the stream function that is described as,

$u=\frac{\partial \psi}{\partial y}, v=-\frac{\partial \psi}{\partial y}$

In terms of these new variables

$u=U_{\infty} f^{\prime}(\eta), v=\frac{1}{2}\left(\frac{v_{n f} U_{\infty}}{x}\right)^{1 / 2}\left(n f^{\prime}(\eta)-f(\eta)\right)$

Finally, we get the similarity results for the velocity and temperature modules as:

$2 L_{0} f^{\prime \prime \prime}+f f^{\prime \prime}=0$

$[1+N r] L_{1} \theta^{\prime \prime}+\frac{(\operatorname{Pr})_{n f}}{2} f \theta^{\prime}+L_{2}(\operatorname{Pr})_{n f}(E c)_{n f} f^{\prime \prime 2}+L_{3} C e^{-n}=0$

The transmuted boundary conditions are found as:

$$
\left.\begin{array}{c}
f(0)=S, f^{\prime}(0)=\lambda, \theta(0)=1 \text { at } \eta=0 \\
f^{\prime}(\eta)=1, \theta(\eta)=0 \quad \text { as } \eta \rightarrow \infty
\end{array}\right\}
$$

where (') represents derivative with respect to $\eta, \lambda$ is the slip velocity term, the term $\mathrm{Nr}$ denotes thermal radiation, Ec represents Eckert number, Pr stands for PrandtI number, and $S$ is the local injection parameter $(<0)$, or the local suction parameter $(>0)$ or these terms are respectively denoted as follows physics validity and the results offered in this study are locally self-sufficient as seen in Sparrow and Yu [29]

Hence

$$
\left.\lambda=A\left(\frac{U_{\infty}^{3}}{v_{n f}}\right)^{1 / 2}, S=B\left(\frac{1}{v_{n f} U_{\infty}}\right)^{1 / 2}\right\}
$$

In addition to that $L_{0}, L_{1}, L_{2}$ and $L_{4}$ are constants which are defined as follows

$$
\begin{gathered}
L_{0}=\frac{1}{(1-\phi)^{2.5}\left[(1-\phi)+\phi\left(\rho_{s} / \rho_{f}\right)\right]}, \quad L_{1}=\frac{k_{n f} / k_{f}}{\left[(1-\phi)+\phi\left(\left(\rho C_{p}\right)_{s} /\left(\rho C_{p}\right)_{f}\right)\right]}, \\
L_{2}=\frac{1}{(1-\phi)^{2.5}\left[(1-\phi)+\phi\left(\left(\rho C_{p}\right)_{s} /\left(\rho C_{p}\right)_{f}\right]\right.}, \quad L_{4}=\frac{\left.1-\phi)+\phi\left(\rho C_{p}\right)_{s} /\left(\rho C_{p}\right)_{f}\right)}{(1-\phi)}
\end{gathered}
$$

In material processing, important engineering design quantities are the skin friction coefficient $C_{f}$ and local Nusselt number $N u_{x}$ are defined as

$C_{f}=\frac{\tau_{w}}{\rho_{n f} U_{\infty}^{2} / 2}, N u_{x}=\frac{x q_{w}}{k_{n f}\left(T_{w}-T_{\infty}\right)}$,

where the shear stress $\tau_{w}=\mu\left(\frac{\partial u}{\partial y}\right)_{y=0}, q_{w}=$ $-\left(k_{n f}+\frac{16 \sigma^{*} T_{\infty}^{3}}{3 k^{*}}\right)\left(\frac{\partial T}{\partial y}\right)_{y=0}$, where $\mu$ is the dynamic viscosity.

The skin friction is taken as,

$N r=\frac{k_{f} k^{*}}{4 \sigma^{*} T_{\infty}^{3}}, E C=\frac{U^{2}}{\left(C_{p}\right)_{f}\left(T_{w}-T_{\infty}\right)},(\operatorname{Pr})_{n f}=\frac{v_{n f}}{\alpha_{n f}}, \lambda=\left(\frac{U_{\infty}^{3}}{x v_{f}}\right)^{1 / 2}, S=\left(\frac{x}{v_{n f} U_{\infty}}\right)^{1 / 2} v_{0}(x)$ 
Table 2 Values of skin friction for various nanofluids

\begin{tabular}{lll}
\hline$\lambda=0, S=0, C=0$ & Nanofluids & \\
\cline { 2 - 3 } & Cu-water & $\mathrm{Al}_{2} \mathrm{O}_{3}$-water \\
\hline $\operatorname{Re}_{x}^{1 / 2} C_{f}$ & & \\
$\phi=0.0$ & 0.3321 & 0.3321 \\
$\phi=0.1$ & 0.403237 & 0.403237 \\
$\phi=0.2$ & 0.5801 & 0.5801 \\
\hline
\end{tabular}

Table 3 Missing slops $f^{\prime \prime}(0)$ and $\theta^{\prime}(0)$ calculation for varying $S$ with constant $\mathrm{Pr}, \mathrm{EC}, \mathrm{Nr}, \lambda$

\begin{tabular}{|c|c|c|c|c|}
\hline \multirow{3}{*}{$\begin{array}{l}\phi=0.1, N r=0.1, \\
\lambda=-0.3, E c=0.1\end{array}$} & \multicolumn{4}{|c|}{ Nanofluids $\left(\mathrm{Al}_{2} \mathrm{O}_{3}\right.$-water) } \\
\hline & \multicolumn{2}{|c|}{$\begin{array}{l}\text { Gangadhar [25] (with- } \\
\text { out IHG) }\end{array}$} & \multicolumn{2}{|c|}{ Present (with IHG) } \\
\hline & $f^{\prime \prime}(0)$ & $\theta^{\prime}(0)$ & $f^{\prime \prime}(0)$ & $\theta^{\prime}(0)$ \\
\hline$S=0.0$ & 0.2524 & -0.1101 & 0.2524 & 0.4869 \\
\hline$S=0.2$ & 0.3788 & -0.4688 & 0.3788 & 0.1968 \\
\hline$S=0.4$ & 0.4974 & -0.9366 & 0.4974 & -0.2346 \\
\hline
\end{tabular}

Table 4 Missing slops $f^{\prime \prime}(0)$ and $\theta^{\prime}(0)$ calculation for varying $S$ with constantPr, Ec, Nr, $\lambda$

\begin{tabular}{|c|c|c|c|c|}
\hline \multirow{3}{*}{$\begin{array}{l}\phi=0.1, \quad N r=0.1, \\
\lambda=-0.3, E c=0.1\end{array}$} & \multicolumn{4}{|c|}{ Nanofluids (Cu-water) } \\
\hline & \multicolumn{2}{|c|}{$\begin{array}{l}\text { Gangadhar [25] (with- } \\
\text { out IHG) }\end{array}$} & \multicolumn{2}{|c|}{ Present (with IHG) } \\
\hline & $f^{\prime \prime}(0)$ & $\theta^{\prime}(0)$ & $f^{\prime \prime}(0)$ & $\theta^{\prime}(0)$ \\
\hline$S=0.0$ & 0.2524 & -0.1734 & 0.2524 & 0.4887 \\
\hline$S=0.2$ & 0.3788 & -0.3358 & 0.3788 & 0.3427 \\
\hline$S=0.4$ & 0.4974 & -0.5056 & 0.4974 & 0.1873 \\
\hline
\end{tabular}

$C_{f} \sqrt{\operatorname{Re}_{x}}=\frac{f^{\prime \prime}(0)}{(1-\phi)^{2.5}}$

For this present paper the skin friction has no impact on the result. Therefore, the focus is on the local Nusselt number,

$N u_{x} \operatorname{Re}_{x}^{-1 / 2}=-\left(\frac{k_{n f}}{k_{f}}+\frac{4}{3 N r}\right) \theta^{\prime}(0)$,

where $\operatorname{Re}_{x}=\left(U_{\infty} x\right) / v$ is the local Reynolds number.
Table 5 Missing slops $f^{\prime \prime}(0)$ and $\theta^{\prime}(0)$ calculation for varying $\lambda$ with constantPr, Ec, Nr, s

\begin{tabular}{|c|c|c|c|c|}
\hline \multirow{3}{*}{$\begin{array}{l}\phi=0.1, N r=1.0, \\
S=0.5, E C=0.1\end{array}$} & \multicolumn{4}{|c|}{ Nanofluids $\left(\mathrm{Al}_{2} \mathrm{O}_{3}\right.$-water) } \\
\hline & \multicolumn{2}{|c|}{$\begin{array}{l}\text { Gangadhar [25] (with- } \\
\text { out IHG) }\end{array}$} & \multicolumn{2}{|c|}{ Present (with IHG) } \\
\hline & $f^{\prime \prime}(0)$ & $\theta^{\prime}(0)$ & $f^{\prime \prime}(0)$ & $\theta^{\prime}(0)$ \\
\hline$\lambda=-0.4$ & 0.3712 & -0.6533 & 0.3712 & -0.2531 \\
\hline$\lambda=0.0$ & 0.5288 & -1.0184 & 0.5288 & -0.6618 \\
\hline$\lambda=0.4$ & 0.5404 & -1.2811 & 0.5404 & -0.9463 \\
\hline
\end{tabular}

Table 6 Missing slops $f^{\prime \prime}(0)$ and $\theta^{\prime}(0)$ calculation for varying $\lambda$ with constantPr, Ec, Nr, s

\begin{tabular}{|c|c|c|c|c|}
\hline \multirow{3}{*}{$\begin{array}{l}\phi=0.1, N r=1.0 \\
S=0.5, E C=0.1\end{array}$} & \multicolumn{4}{|c|}{ Nanofluids (Cu-water) } \\
\hline & \multicolumn{2}{|c|}{$\begin{array}{l}\text { Gangadhar [25] (with- } \\
\text { out IHG) }\end{array}$} & \multicolumn{2}{|c|}{ Present (with IHG) } \\
\hline & $f^{\prime \prime}(0)$ & $\theta^{\prime}(0)$ & $f^{\prime \prime}(0)$ & $\theta^{\prime}(0)$ \\
\hline$\lambda=-0.4$ & 0.3712 & -0.2531 & 0.3712 & 0.0206 \\
\hline$\lambda=0.0$ & 0.5288 & -0.6618 & 0.5288 & -0.1716 \\
\hline$\lambda=0.4$ & 0.5404 & -0.9463 & 0.5404 & -0.3100 \\
\hline
\end{tabular}

\section{Numerical procedure}

The coupled system of Eqs. (10) and (11) in combination with conditions (12) was numerically solved using the bvp4c package from Matlab. The package is built on boundary value procedures. The conditions (12) are substituted by utilizing the similarity variable $\eta_{\max }$ of value 8 which is taken at the infinity $(\infty)$ value (assumed) of $\eta$. The chosen of $\eta_{\max }=8$ shows that the far stream boundary conditions satisfies correctly. Solutions for the values of $\mathrm{Ec}, \mathrm{Pr}, \mathrm{Nr}, \mathrm{C}$ and $\mathrm{S}$ are nevertheless suitable since they demonstrate the core features of the response boundary layers. Here we have taken the values of Prandtl number, $\operatorname{Pr}=2.37$ (for copper water nanofluid), $\operatorname{Pr}=6.38$ (for alumina water nanofluid). Eckert number, $E c=0.5,1.0$, radiation parameter, $\mathrm{Nr}=0.0,1.0,2.0, \mathrm{C}=0.0$ (for without heat generation), $C=1.0$ (for heat generation), suction parameter, $S=0.0,0.2,0.4$ and velocity ratio parameter, $\lambda=-0.4,0.0,0.4$. 
Table 7 Missing slops $f^{\prime \prime}(0)$ and $\theta^{\prime}(0)$ calculation for varying $E c$ with constant $\mathrm{Pr}, \lambda, \mathrm{Nr}, \mathrm{s}$

\begin{tabular}{|c|c|c|c|c|}
\hline \multirow{3}{*}{$\begin{array}{l}N r=1.0, S=0.5 \\
\lambda=-0.35\end{array}$} & \multicolumn{4}{|c|}{ Nanofluids $\left(\mathrm{Al}_{2} \mathrm{O}_{3}-\right.$ water $)$} \\
\hline & \multicolumn{2}{|c|}{$\begin{array}{l}\text { Gangadhar [25] (with- } \\
\text { out IHG) }\end{array}$} & \multicolumn{2}{|c|}{ Present (with IHG) } \\
\hline & $f^{\prime \prime}(0)$ & $\theta^{\prime}(0)$ & $f^{\prime \prime}(0)$ & $\theta^{\prime}(0)$ \\
\hline$E C=0.1$ & 0.5506 & -1.1059 & 0.5506 & 0.1539 \\
\hline$E C=0.5$ & 0.5506 & -0.2404 & 0.5506 & 0.2404 \\
\hline$E C=1.0$ & 0.5506 & 0.8415 & 0.5506 & 0.8415 \\
\hline
\end{tabular}

Table 8 Missing slops $f^{\prime \prime}(0)$ and $\theta^{\prime}(0)$ calculation for varying $E c$ with constantPr, $\lambda, N r, s$

\begin{tabular}{|c|c|c|c|c|}
\hline \multirow{3}{*}{$\begin{array}{l}N r=1.0, S=0.5 \\
\lambda=-0.35\end{array}$} & \multicolumn{4}{|c|}{ Nanofluids (Cu-water) } \\
\hline & \multicolumn{2}{|c|}{$\begin{array}{l}\text { Gangadhar [25] (with- } \\
\text { out IHG) }\end{array}$} & \multicolumn{2}{|c|}{ Present (with IHG) } \\
\hline & $f^{\prime \prime}(0)$ & $\theta^{\prime}(0)$ & $f^{\prime \prime}(0)$ & $\theta^{\prime}(0)$ \\
\hline$E C=0.1$ & 0.5506 & -0.5551 & 0.5506 & -0.5551 \\
\hline$E C=0.5$ & 0.5506 & -0.2426 & 0.5506 & 0.4664 \\
\hline$E C=1.0$ & 0.5506 & 0.1481 & 0.5506 & 0.8570 \\
\hline
\end{tabular}

Table 9 Missing slops $f^{\prime \prime}(0)$ and $\theta^{\prime}(0)$ calculation for varying $N r$ with constant $\operatorname{Pr}, \lambda, s$

\begin{tabular}{|c|c|c|c|c|}
\hline \multirow{3}{*}{$\begin{array}{l}E c=0.1, S=0.5, \\
\lambda=-0.3\end{array}$} & \multicolumn{4}{|c|}{ Nanofluids $\left(\mathrm{Al}_{2} \mathrm{O}_{3}\right.$-water) } \\
\hline & \multicolumn{2}{|c|}{$\begin{array}{l}\text { Gangadhar [25] (with- } \\
\text { out IHG) }\end{array}$} & \multicolumn{2}{|c|}{ Present (with IHG) } \\
\hline & $f^{\prime \prime}(0)$ & $\theta^{\prime}(0)$ & $f^{\prime \prime}(0)$ & $\theta^{\prime}(0)$ \\
\hline$N r=0.1$ & 0.5506 & -1.1059 & 0.5506 & -0.3722 \\
\hline$N r=1.0$ & 0.5506 & -1.2097 & 0.5506 & -0.3878 \\
\hline$N r=2.0$ & 0.5506 & -1.5509 & 0.5506 & -0.4090 \\
\hline
\end{tabular}

\section{Results and discussion}

After solving the governing system of equations numerically, the results obtained are illustrated in figures and tables. The influence of various dimensionless terms on the linear velocity and energy components is demonstrated. Computed results for the skin friction and heat gradient number in the considered nanofluids (alumina and copper liquid) are presented in tables. The missing slops for varying parameters are also calculated for various examined nanofluids and compared with previous studies.
Table 10 Missing slops $f^{\prime \prime}(0)$ and $\theta^{\prime}(0)$ calculation for varying $\mathrm{Nr}$ with constant $\operatorname{Pr}, \lambda$, $s$

\begin{tabular}{|c|c|c|c|c|}
\hline \multirow{3}{*}{$\begin{array}{l}E c=0.1, S=0.5 \\
\lambda=-0.3\end{array}$} & \multicolumn{4}{|c|}{ Nanofluids (Cu-water) } \\
\hline & \multicolumn{2}{|c|}{$\begin{array}{l}\text { Gangadhar [25] (with- } \\
\text { out IHG) }\end{array}$} & \multicolumn{2}{|c|}{ Present (with IHG) } \\
\hline & $f^{\prime \prime}(0)$ & $\theta^{\prime}(0)$ & $f^{\prime \prime}(0)$ & $\theta^{\prime}(0)$ \\
\hline$N r=0.1$ & 0.5506 & -0.5551 & 0.5506 & 0.1539 \\
\hline$N r=1.0$ & 0.5506 & -0.7025 & 0.5506 & 0.1092 \\
\hline$N r=2.0$ & 0.5506 & -0.8322 & 0.5506 & 0.0964 \\
\hline
\end{tabular}

(a)
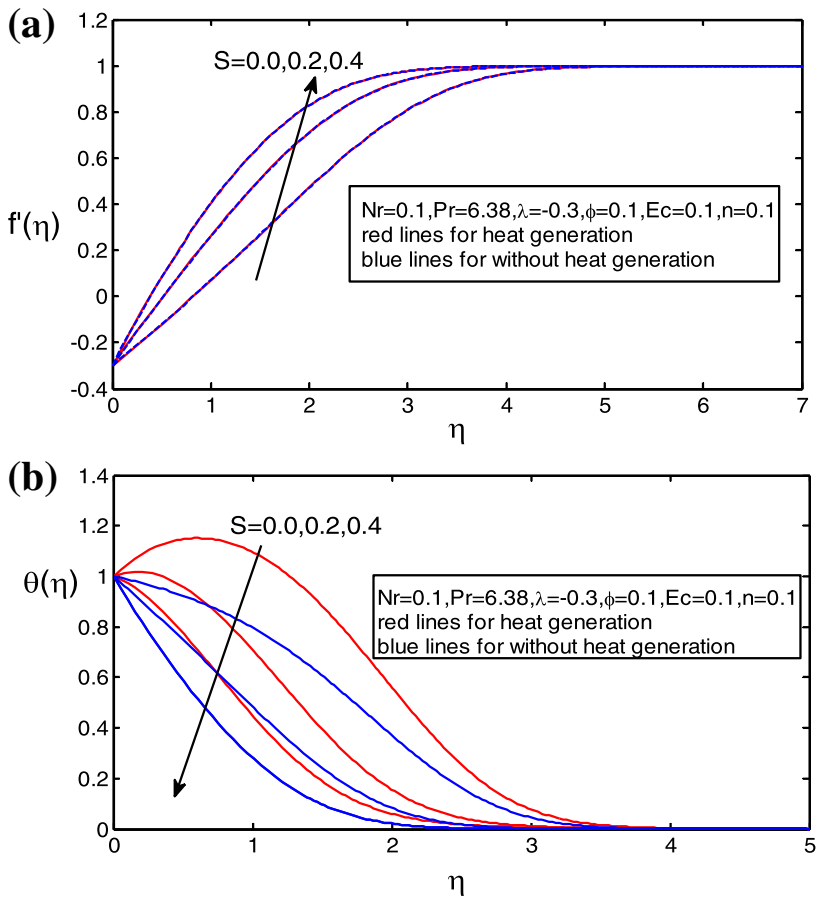

Fig. 2 a velocity behaviour for $S$ with Alumina-Water for $N r=0.1, \lambda=-0.3$ b Temperature behaviour for $S$ with AluminaWater for $\mathrm{Nr}=0.1, \lambda=-0.3$

In Table 2, the computed results for the skin friction is presented for different nanofluids. As shown is the table, the skin friction coefficient has no substantial influence on the variation of Prandtl number, but the Nusselt number shows an impactful effect. More also, the computed values are verified for $f^{\prime \prime}(0)=0.332$ at $\phi=0, \lambda=0$ and $S=0$ which is found to be in agreement with what was obtained in other studies. Hence, our presented results are valid for further study.

From Tables 3 and 4, the computational results for the missing slops is depicted for the heat gradient $\theta^{\prime}(0)$ and nanofluids wall effect $f^{\prime \prime}(0)$ for different parameter values. 
It is seen that the values of the missing slope for $\theta^{\prime}(0)$ is decreasing while $f^{\prime \prime}(0)$ is increasing as the values of the term suction $(S)$ rises for both 'Alumina water and Copper water'. Variation of suction number shows no momentous effect for $f^{\prime \prime}(0)$ in both nanofluids considered. However, the decreasing rate of $\theta^{\prime}(0)$ is slower in alumina water than copper water nanofluids. These agreed well with the report of Gangadhar [16].

In Tables 5 and 6, the computed values for the missing slops is presented for the Nusselt number $\theta^{\prime}(0)$ and skin friction $f^{\prime \prime}(0)$ for constant values of terms $\mathrm{Pr}, E c, N r, \lambda$. As observed in the tables, the heat gradient is decreasing while the nanofluid wall friction is increasing as the values of velocity slip $(\lambda)$ is increased for both 'Alumina water and Copper nanoliquids'. Meanwhile, varying of velocity slip shows no substantial effect for $f^{\prime \prime}(0)$ in the two cases of the examined nanofluids. Therefore, diminishing rate of $\theta^{\prime}(0)$ is lesser for copper water than alumina water nanofluid as obtained from the investigation.

From Tables 7 and 8 , the impact of variation in the term $E c$ on $\theta^{\prime}(0)$ and $f^{\prime \prime}(0)$ is numerically demonstrated for some fixed parameter values. The value of $\theta^{\prime}(0)$ is increasing and $f^{\prime \prime}(0)$ decreasing for the different values of $E c$ for both 'Alumina and Copper Nano-water', this is found to be in alliance with the work of Gangadhar [16]. For varying Eckert number (Ec), the impact is not
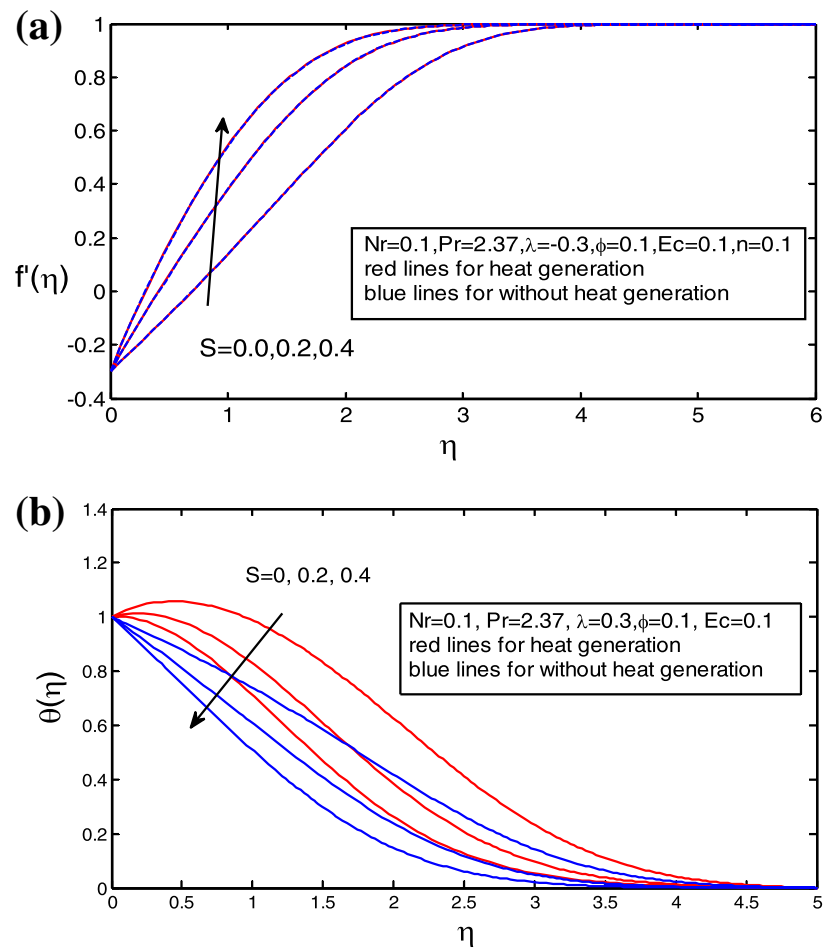

Fig. 3 a Velocity behaviour for $S$ with Copper-Water for $N r=0.1, \lambda=-0.3$ b. Temperature behaviour for $S$ with CopperWater for $\mathrm{Nr}=0.1, \lambda=-0.3$
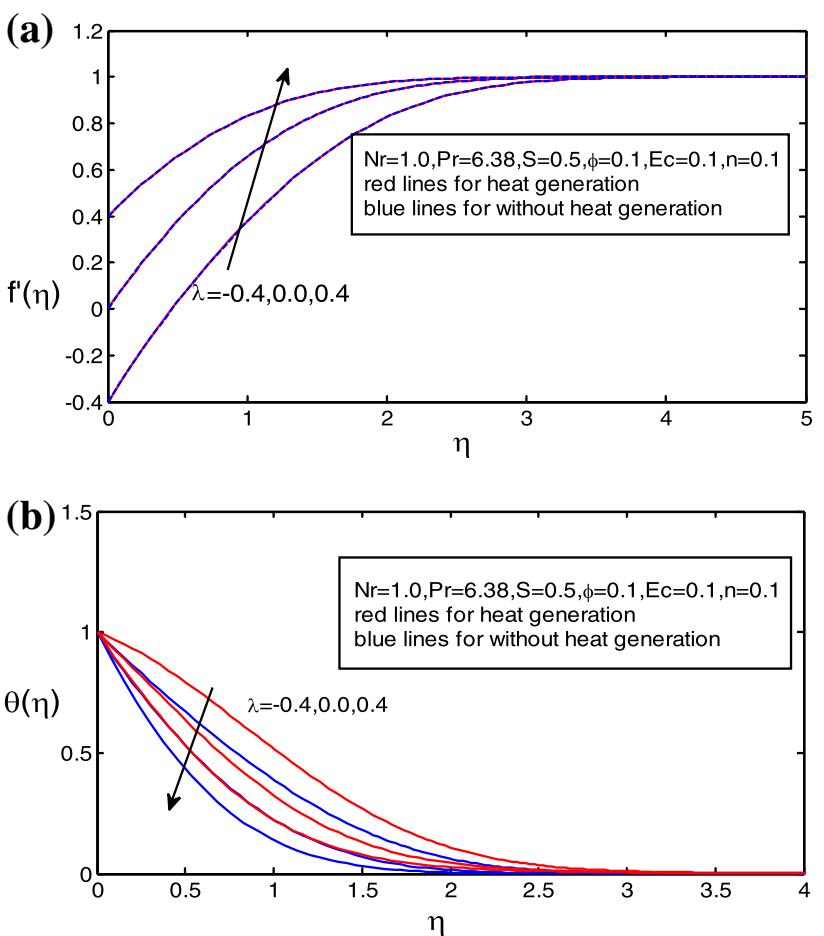

Fig. 4 a Velocity behaviour for $\lambda$ with Alumina-Water for $N r=1.0, S=0.5, E c=0.1 \mathbf{b}$ Temperature behaviour for $\lambda$ with Alumina-Water for $\mathrm{Nr}=1.0, \mathrm{~S}=0.5, \mathrm{EC}=0.1$

significant for $f^{\prime \prime}(0)$ as depicted in the tables. Therefore, increasing rate of $\theta^{\prime}(0)$ is reduced for copper nanowater than alumina nano-water. Hence, Nano-structure is enhanced in the alumina nanofluid than copper nanofluid.

Tables 9 and 10 show the calculated values for missing slopes with varying values of the term $\mathrm{Nr}$ in the physical wall effect $f^{\prime \prime}(0)$ and $\theta^{\prime}(0)$. Evaluation of $\theta^{\prime}(0)$ shows a decreasing in the numerical values, but $f^{\prime \prime}(0)$ is increasing as the values of radiation $(\mathrm{Nr})$ is encouraged for both 'Alumina and Copper Nano-liquid. Variation of radiation number has no influence on $f^{\prime \prime}(0)$ as presented in the tables. Here, decreasing rate of $\theta^{\prime}(0)$ is reduced for copper nanofluid than alumina nanoliquid. This is in agreement with the report of a reference [16] in the absence of heat generation.

Figure $2 \mathrm{a}$ and $\mathrm{b}$ depict the reaction of alumina water nanofluid flow rate and energy distribution in a horizontal motioning plate with different values of suction (S). As obtained from the graphs, the velocity field of the alumina nano-water is encouraged as the suction term values is increased as presented in the Fig. 2a. The rise in the flow magnitude is due to an increase in the nanoparticles colloidal suspension that enhanced the particle dispersion of alumina water. The nanofluid mixture does not settle, this leads to free collision of nanoparticles that in turn raised 

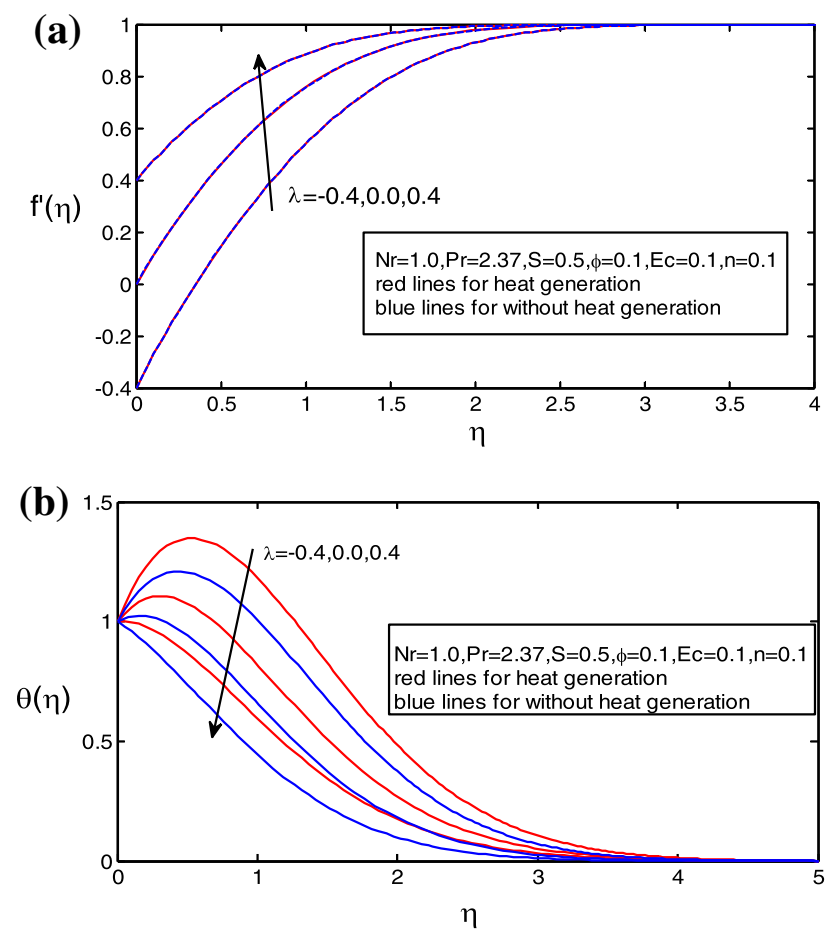

Fig. 5 a Velocity behaviour for $\lambda$ with Copper-Water with $\mathrm{Nr}=1.0, S=0.5, E C=0.1 \mathbf{b}$ Temperature behaviour for $\lambda$ with Copper-Water for $\mathrm{Nr}=1.0, \mathrm{~S}=0.5, \mathrm{EC}=0.1$

the velocity profile. In Fig. $2 b$, either in the existence or absence of heat generation, it is seen that the heat field magnitude reduces. The diminishing in the overall energy dispersion is as a result of thinner in the energy boundary layer that causes more heat to leave the system. This resulted in a decline in the quantity of heat transfer in the flow medium, the outcome supported the claim by Amanullah et al. [11]. Figure $3 a$ and $b$ for the flow field and heat distribution demonstrate the same behaviour for copper water nanofluid with an increasing suction term (S). The flow velocity rises in the presence of heat generation, this is because the fluid temperature increases as the soluble nanofluids mixture reacted which breaks the fluid bonding force and cause the nanoparticle to move freely. Therefore, the nanofluid flow rate increases in the flow medium due to reduction in the fluid viscosity resulting in free flow of the fluid. Meanwhile, the temperature distribution within the system is discouraged as more heat diffused out of the colloidal suspension mixture. This is possible due to diminish in the heat limiting layer that leads to decline in the energy transfer component as seen in Fig. 3b. An increase in the overall heat transfer to the surroundings assists in reducing the quantity of heat within the nanofluid reaction system. Hence, this prevent excessive heat that can cause blow up and low performance of industrial thermal
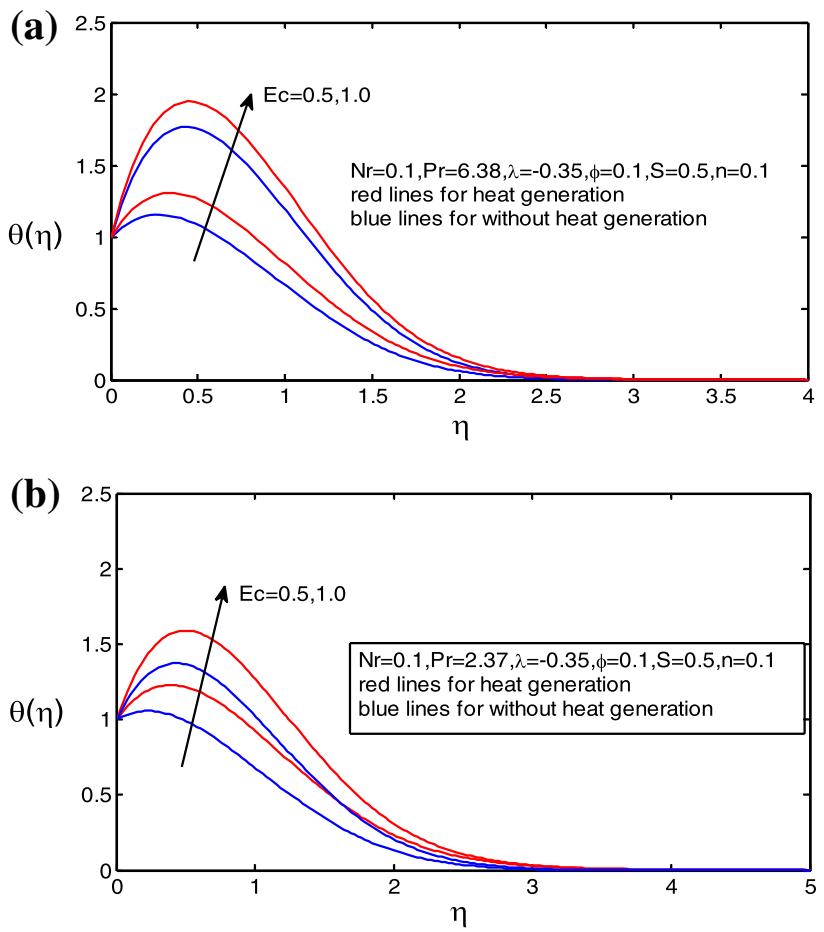

Fig. 6 a Temperature behaviour for Ec with Alumina-Water for $N r=0.1, S=0.5, \lambda=-0.35 \mathbf{b}$ Temperature behaviour for $E c$ with Copper-Water for $\mathrm{Nr}=0.1, S=0.5, \lambda=-0.35$
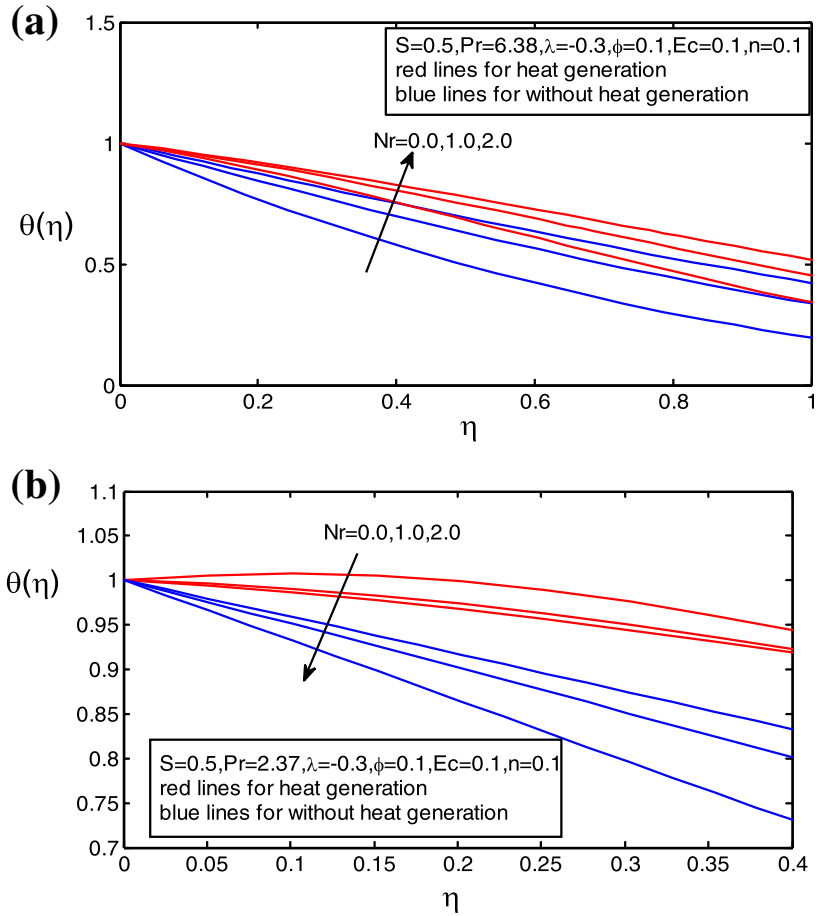

Fig. 7 a Temperature behaviour for $N r$ with Alumina-Water for $E c=0.1, S=0.5, \lambda=-0.3$. b Temperature behaviour for $\mathrm{Nr}$ with Copper-Water for $E c=0.1, S=0.5, \lambda=-0.3$ 


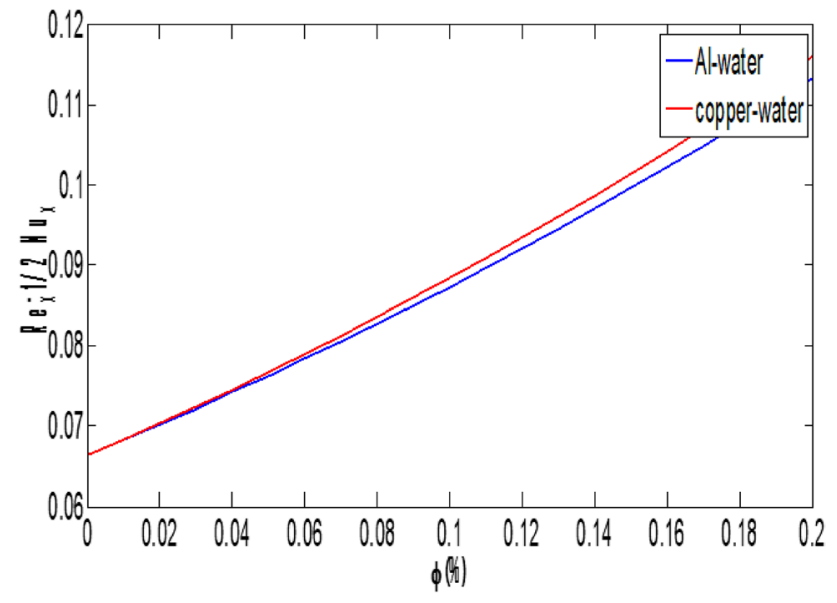

Fig. 8 The Impact of volume Fraction $\phi$ in Nusselt number for Alumina-water nanofluids and Copper-water nanofluids

technology, this agreed well with what was reported by Okedoye and Salawu [30].

The response of the alumina nanofluid velocity and heat field to rising in the velocity slip term $(\lambda)$ are illustrated in the Figs. $4 a$ and b. As the slip velocity of the porous plate is increased, the nanofluid velocity profile momentously rises close to the moving plate, but the flow rate gets reduced as it moves steadily away from the moving surface to the far boundary layer. This is normal since the considered nanofluids flow is only being propelled by the moving surface as no pressure or thermal convection exists in the system. Nevertheless, the temperature field is discouraged as depicted in Fig. $4 \mathrm{~b}$. As the slip velocity is raised, a noteworthy reduction in the temperature is noticed close to the slip wall. This is as a result of heat being released through the porous plate, its decreases gradually further away from the surface until it becomes uniform in the system as achieved in [17, 22]. Similarly, copper nanofluid exhibited the same characteristic for the heat and velocity components as represented in Fig. $5 \mathrm{a}$ and b. In Fig. 5a, the fluid thermal conductivity is enhanced that in turn discourages the copper liquid bonding force. This allows free nanoparticles motion in the mixture that leads to a boost in the velocity magnitude as the slip term is increased. Therefore, the parameter will assist in raising industrial fluid flow in order to enhance productivity. Meanwhile, with or without internal heat generation the copper nanofluid temperature decreases regularly until it attains a uniform heat distribution as observed in Fig. 5b. Thus, more heat diffusion occurs near the slip porous wall due to shrinking in the heat boundary viscosity. However, parameter that decrease heat production in a nanofluid mixture should be encouraged because it will support and help in maintaining viscoelastic material viscosity as reported by Salawu et al. [31].
Figure $6 \mathrm{a}$ and $\mathrm{b}$ depict respectively, the heat profiles for the rising effect of heat dissipation term $(\mathrm{Ec})$ on the alumina and copper water nanofluid temperature dispersion. As seen, the heat component increases for both considered nanoliquids. The effect is significant near the velocity slip plate, this is due to the difference between the boundary layer enthalpy and the nanofluids kinetic energy. The local specific heat is enhanced as the variance between the local heat and plate heat is increased. Hence, this enhanced the thickness of the boundary layer that resulted in high heat distribution in the nano-species mixture. Therefore, the temperature profiles is encouraged. Moreover, rising in the radiation term $(\mathrm{Nr})$ values causes overall reduction in the alumina and copper water temperature fields as presented in Fig. 7a and b. The decreasing effect is lowered close to the slip plate, but reduces progressively as it travels towards the far stream layer. The release of energy to the surroundings is strengthened as moving subatomic nanoparticles carries heat from the slip plate to the far boundary layer. Thus, the magnitude of temperature distribution is generally discouraged in the nanoliquids as the values of $(\mathrm{Nr})$ is raised. The influence of volume friction in wall coefficient heat transfer (Nusselt number) is demonstrated in Fig. 8 for the alumina nanoliquid and copper water nanoliquid. The plot shows the temperature gradient for the nanofluid volume friction at the plate surface.

\section{Conclusions}

In the present study, a detailed mathematical formulation of incompressible fluid has been offered for steadystate heat transport boundary layer flow of a nanofluid past a flat motioning plate, stimulated by nanomaterial coating applications. Two different nanoparticles $(\mathrm{Cu}, \mathrm{Al})$ have been examined to simulate nanoscale impacts. The radiation and IHG influences have been included. With applicable similarity quantities for the momentum and energy transformation, the main dimensional model has been changed to a couple of ordinary derivative equations. These equations under appropriate wall and free stream boundary conditions have been computationally solved through the MATLAB package bvp4c. Validation of solutions with earlier distinct case of the overall model available in the literature has been performed. A detailed analysis of the impact of suction, velocity slip, Eckert number and thermal radiation on the momentum and thermal characteristics (including coefficient of skin dragging and energy gradient) has been conducted. The present numerical simulations have shown that: 
- The rates of change of velocity in both water-based nanoparticles are aided with improving values of $S, \lambda$. Whereas reverse orientation is perceived in rates of change of temperature.

- Increasing values of the $E c$, raises the temperature changes rate in both water-based nanoparticles are intensified.

- Rising values of the $\mathrm{Nr}$, increases the temperature changes rate for both water-based nanoparticles are intensified.

- Nusselt number accelerates as nanoparticle volume fraction for both water-based nanoparticles raise.

It is predicted that this analysis carried out here will motivate additional attention in more realist industrial electro conductive magneto nano-polymers. The present investigation is valid for nanofluid flow of free convection over a flat plate, complex geometries mixed convective flow including non-Newtonian fluids like Casson, Williamson and Sisko fluid are underway and will be communicated imminently. The study is limited to heat transfer of nanofluid without considering chemical reaction.

Acknowledgements The authors really appreciate the suggestions and comments of the reviewers which are served to increase the quality of the current analysis.

\section{Compliance with ethical standards}

Conflict of interest The authors declare that they have no conflict of interest.

Availability of data material All data is available with authors only.

Open Access This article is licensed under a Creative Commons Attribution 4.0 International License, which permits use, sharing, adaptation, distribution and reproduction in any medium or format, as long as you give appropriate credit to the original author(s) and the source, provide a link to the Creative Commons licence, and indicate if changes were made. The images or other third party material in this article are included in the article's Creative Commons licence, unless indicated otherwise in a credit line to the material. If material is not included in the article's Creative Commons licence and your intended use is not permitted by statutory regulation or exceeds the permitted use, you will need to obtain permission directly from the copyright holder. To view a copy of this licence, visit http://creativecommons .org/licenses/by/4.0/.

\section{References}

1. Chmielewski AG, Haji-Saeid M, Ahmed S (2005) Progress in radiation processing of polymers. Nucl Instrum Methods Phys Res Sect B 236:44-54

2. Bergman TL, Viskanta R (1996) Radiation heat transfer in manufacturing and materials processing. In: Menguc MP (ed) Radiative Transfer-I. Begell House Publishing, New York, pp 13-39
3. Shamshuddin MD, Mishra SR, Anwar Beg O, Kadir A (2019) Adomain decomposition method simulation of Von Karman swirling bioconvection nanofluid flow. J Central South Univ 26(10):2797-2813

4. Kadir A, Anwar Beg O, El Gendy M, Beg TA, Shamshuddin MD (2019) Computational fluid dynamic and thermal stress analysis of coating for high temperature corrosion protection of Aerospace gas turbine blades. Heat Transf Asian Res 48:2302-2328

5. Liu B, Li BQ, Li Z, Bai P, Wang Y, Kuai Z (2019) Numerical investigation on heat transfer of multi-laser processing during selective laser melting of ALSi10Mg. Results Phys 12:454-459

6. Yue Z, Reitz RD (2019) Numerical investigation of radiative heat transfer in internal combustion energies. Appl Energies 235:147-163

7. Choi SUS, Zhang ZG, Yu W, Lockwood FE, Grulke EA (2001) Anomalous thermal conductivity enhancement in nanotube suspensions. Appl Phys Let 79(14):2252-2254

8. Masuda H, Ebata A, Teramae K (1993) Alteration of thermal conductivity and viscosity of liquid by dispersing ultra-fine particles. Dispersion of $\mathrm{Al}_{2} \mathrm{O}_{3}, \mathrm{SiO}_{2}$ and $\mathrm{TiO}_{2}$ ultra-fine particles. Netsu Bussei 7:227-233

9. Hamid M, Zubair T, Usman M, Haq RU (2019) Numerical investigation of fractional-order unsteady natural convective radiating flow of nanofluid in a vertical channel. AIMS Math 4(5):1416-1429

10. Pandey AK, Kumar M (2017) Boundary layer flow and heat transfer analysis on $\mathrm{Cu}$-water nanofluid flow over a stretching cylinder with slip. Alex Eng J 56(4):671-677

11. Amanullah Ch, Nagendra N, Reddy MSN, Rao AS, Anwar Beg O (2017) Mathematical study of non-Newtonian nanofluid transport phenomena from an isothermal sphere. Front Heat Mass Transfer 8:29. https://doi.org/10.5098/hmt.8.29

12. Anwar Bég O, Sanchez Espinoza DE, Kadir A, Shamshuddin MD, Ayesha S (2018) Experimental study of improved rheology and lubricity of drilling fluid enhanced with nano-particles. Appl Nanosci 8(5):1069-1090

13. Sheikholeslami M, Shamlooei M, Moradi R (2018) Numerical simulation for heat transfer intensification of nanofluid in a permeable curved enclosure considering shape effect of $\mathrm{Fe}_{3} \mathrm{O}_{4}$ nanoparticles. Chem Eng Proc Proc Intensification 124:71-82

14. Shamshuddin MD, Mishra SR, Anwar Beg O, Kadir A (2018) Unsteady Chemo-Tribological Squeezing Flow of Magnetized Bioconvection Lubricants: Numerical Study. J Nanofluids 8(2):407-419

15. Anwar Beg O, Kuharat S, Ferdows M, Das M, Kadir A, Shamshuddin MD (2019) Modelling magnetic nanopolymer flow with induction and nanoparticle solid volume fraction effects: solar magnetic nanopolymer fabrication volume fraction. IMechE Part N. J Nanomater Nanoeng Nanosys 233(1):27-45

16. Rashidi S, Masoud B, Esfahani JA, Ahmadi G (2016) Discrete particle model for convective $\mathrm{Al}_{2} \mathrm{O}_{3}$-water nanofluid around a triangular obstacle. Appl Therm Eng 100:39-54

17. Akar S, Rashidi S, Esfahani JA (2018) Second law of thermodynamic analysis for nanofluid turbulent flow around a rotating cylinder. JTherm Analys Calorimetry 132:1189-1200

18. Shamsabadi H, Rashidi S, Esfahani JA (2019) Entropy generation analysis for nanofluid flow inside a duct equipped with porous baffles. J Therm Analys Calorimetry 135:1009-1019

19. Eid MR (2020) Thermal characteristics of 3D nanofluid flow over a convectively heated Riga surface in a Darcy-Forchheimer porous material with linear thermal radiation: an optimal analysis. Arabian J Sci Eng. https://doi.org/10.1007/s13369-020-04943 $-3$

20. Eid MR, Mabood F (2020) Two-phase permeable non-Newtonian cross-nanomaterial flow with Arrhenius energy and entropy 
generation: Darcy-Forchheimer model. Physica Scripta. https ://doi.org/10.1088/1402-4896/abb5c7

21. Eid MR, Mabood F (2020) Entropy analysis of a hydromagnetic micropolar dusty carbon NTs-kerosene nanofluid with heat generation: Darcy-Forchheimer scheme. J Therm Analys Calorimetry. https://doi.org/10.1007/s10973-020-09928-w

22. Mohebbi R, Rashidi MM, Izadi M, Sidik NAC, Xian HW (2018) Forced convection of nanofluids in an extended surfaces channel using Lattice Boltzmann method. Int J Heat Mass Transfer 117:1291-1303

23. Mohebbi R, Rashidi MM (2017) Numerical simulations of natural convection heat transfer of a nanofluid in an L-shaped enclosure with a heating obstacle. J Taiwan Inst Chem Eng 72:70-84

24. Armaghani T, Kasaeipoor A, Alavi N, Rashidi MM (2016) Numerical investigation of water-alumina nanofluid natural convection heat transfer and entropy generation in a baffled L-shaped cavity. J Mol Liq 223:243-251

25. Gangadhar K (2016) Radiation and viscous dissipation effects on laminar boundary layer flow nanofluid over a vertical plate with a convective surface boundary condition with suction. J Appl Fluid Mech 9(4):2097-2103
26. Ferdows M, Murtaza MG, Shamshuddin MD (2019) Effect of internal heat generation on free convective power law variable temperature past a vertical plate considering exponential variable viscosity and thermal conductivity. J Egyptian Math Soc 27:56. https://doi.org/10.1186/s42787-019-0062-5

27. Anjali Devi SP, Julie A (2011) Laminar boundary layer flow of nanofluid over a flat plate. Int J Appl Math Mech 7(6):52-71

28. Brewster MQ (1992) Thermal radiative transfer properties. Wiley, New York

29. Sparrow EM, Yu HS (1971) Local non-similarity thermal boundary layer solutions. ASME J Heat Transfer 93(4):328-334

30. Okedoye AM, Salawu SO (2019) Unsteady oscillatory MHD boundary layer flow past a moving plate with mass transfer and binary chemical reaction. SN Appl Sci 1(9):1586

31. Salawu SO, Disu AB and Dada MS (2020) On criticality for a generalized Couette flow of a branch-chain thermal reactive thirdgrade fluid with Reynolds viscosity model. The Scientific World Journal, 20, 10pages

Publisher's Note Springer Nature remains neutral with regard to jurisdictional claims in published maps and institutional affiliations. 